\title{
AN AHP-ISM APPROACH FOR CONSIDERING PUBLIC PREFERENCES IN A PUBLIC TRANSPORT DEVELOPMENT DECISION
}

\author{
Szabolcs DULEBA* \\ Dept of Transport Technology and Economics, Budapest University of Technology and Economics, Hungary
}

Received 24 October 2017; revised 1 March 2018; accepted 17 July 2018

first published online 18 March 2019

\begin{abstract}
Recently, there has been a transparent need to involve public in transport development decisions not only in the EU but also in other countries worldwide. Public involvement in decision-making, however, suffers from two critical issues: lack of expertise and lack of enthusiasm. This paper aims to overcome the first problem: how to amend passenger preferences related to public transport development with expert knowledge on transport systems. For this purpose, a new research methodology has been created which combines the well proven Analytic Hierarchy Process (AHP) and Interpretive Structural Modelling (ISM) methods in a novel way. ISM is used to reveal the non-hierarchical connections of the transport system elements and by this, AHP results are modified with the consideration of element interactions. The first stage of the three-stage-survey has been conducted in Yurihonjo (Japan), the second and third in an international workshop with the participation of experts. Results show that the original AHP scores - gained from passenger evaluations - are significantly modified by adding expert knowledge on factor interactions, thus new preference order is gained related to the importance of the development of public transport system elements. The introduced procedure can be applied for other public transport system improvement decision-making situations in which passenger involvement is required.
\end{abstract}

Keywords: AHP, ISM, passenger preferences, MCDM, element interactions, public involvement.

\section{Introduction}

The recent decades have shown the growing importance of public engagement in transportation decision-making. The notion itself can be defined as "involvement in decision-making with the purpose of influencing the choice(s) being made" (Renn et al. 1995). Public participation has not only become part of legislation - e.g. the Safe, Accountable, Flexible, Efficient Transportation Equity Act: a Legacy for Users - US Government (2005) - but the amount of case studies attempting to solicit public opinion has also been increasing (see among others the developments described by Aparicio (2007); Cascetta and Pagliara (2013) and De Luca (2014). The number of connected guidelines and booklets are also growing - e.g. A Guide to Transportation Decisionmaking - US DoT (2015). Burall and Shahrokh (2010) present nine recommendations on how to engage the public in these decision making processes; among others also declaring that "government must take final responsibility for making fair and balanced policy decisions that are informed by dialogue with the public".

Nevertheless, it still remains difficult to obtain proper information regarding the public opinion of development issues (Jen et al. 2011). Public transportation users have little time or enthusiasm for filling out long questionnaires and do not have the expertise that is necessary for complex and consistent evaluations of the system's elements. Moreover, these elements interact in various ways that make the overall decision very complicated. Finally, the decision-making procedure is long and demanding.

This problem became even more tangible when the public transport situation in Yurihonjo (Japan) was discovered. As passenger numbers had steadily been decreasing, decision makers were actively looking for a way to solicit public opinion and redesign the public transport system with due attention to that. This constituted a direct incentive for elaborating and fine-tuning the Analytic Hierarchy Process (AHP) and Interpretive Structural Modelling (ISM) approach (AHP-ISM).

The initial project idea was to gain information from public transport users by a traditional or slightly modified AHP survey. Having conducted that, however, the representatives of the city public transport company realized that the results did not reflect the influence of system

*E-mail: duleba.szabolcs@mail.bme.hu 
elements on each other so the AHP results showed false ranking, not optimal regarding the ultimate objectives of the decision problem (being the need to determine the exact public preferences for the development of the transport system). Thus, a new procedure had to be elaborated in order to also involve system characteristics and interdependencies of the transport elements.

This paper describes the three-stage AHP-ISM procedure that has been created for solving the above-mentioned problem. At the first level of the new approach, AHP is applied to obtain a consistent opinion on the importance of element improvements (within the tolerance level of inconsistency). Then for considering non-hierarchical connections of the elements, ISM is employed. The third stage introduces modifications of the AHP scoring process by considering the impacts of each element on the other elements.

\section{Literature review}

Due to the complexity of the topic, a clear and structured method is needed, which is easy to understand, simplifies the evaluators' job as much as possible, and remains consistent (Saaty 1977). Fulfilling these criteria, AHP, a well-known Multi-Criteria Decision-Making (MCDM) method (Bhushan, Ria 2004; Barić et al. 2016; Magginas et al. 2018; Al-Atawi et al. 2016; Chen et al. 2016; VitićĆetković, Bauk 2014) was considered. AHP was applied several times in the literature to rank public transport projects. Tudela et al. (2006) used AHP to rank urban transport projects based on their estimated economic and environmental impacts. The authors drew the following two conclusions: when considering urban transport projects, economic and non-economic factors should be included and public opinion should be considered explicitly in decision-making. Another transport system analysis and project selection from an environmental point of view by AHP was conducted by Yedla and Shrestha (2003).

Apart from the advantages of AHP, however, one disadvantage must be emphasized: AHP is only able to consider the hierarchical connections of the examined elements, and it neglects all other relationships. Experience and analysis of real-life situations show, that excluding non-hierarchical relationships leads to an overly simplified presentation of the decision problem, where nonhierarchical connections are often present (Saaty 2013; Jharkharia, Shankar 2007). For example, in the hierarchical structure of a public transportation system, there might be no direct relationship between journey time and physical comfort, but if they are logically considered, it can be seen, that journey time does indeed influence the physical comfort of the passengers. The widely-known Analytic Network Process (ANP) created by Saaty (2001) could not be used due to its complexity considering passenger evaluators.

That was the reason behind the need to create an alternative model that uses the AHP structure but is capable of dealing with non-hierarchical impacts and modifies the final AHP scoring. This is the point where ISM was considered.

Generally, ISM is selected for analysing the interdependencies among the elements of a system. ISM is a well-validated method (Malone 1975) that was created to determine the relationships between the elements of a complex system. Following its first publication, ISM has been used in many scientific publications (Eswarlal et al. 2011; Pfohl et al. 2011). So, as a means of establishing the presence of non-hierarchical connections, ISM is used in the second stage of research. During this phase, connections are gained and the transitivity of the connections is calculated. Transitivity is to be determined to ensure the coherence of the results. Namely, if element $e_{1}$ affects $e_{2}$, and $e_{2}$ affects $e_{3}$, then $e_{1}$ should influence $e_{3}$ as well, and this transitivity is to be duly reflected in the numerical data.

There are several AHP-ISM applications in the scientific literature (e.g. Kannan et al. 2008; Saleeshya et al. 2012; Duleba et al. 2013). The dominant majority of these papers applied ISM for determining the hierarchical structure of the factors in the decision system based on their influential power and then used this hierarchy in the traditional AHP process for determining the importance ranking of the elements in the final decision (Kannan et al. 2008; Saleeshya et al. 2012) applied AHP at the first level of the decision hierarchy and ISM at the second level with the purpose of ranking the elements by their importance of improving the agility of the supply chain in the Indian textile industry. AHP was capable of determining the relative importance of the elements at the first level, but at the second level, the authors elicited the importance of the respective elements based on their dependencies, thus, on their influence on each other. Nevertheless, none of these techniques can simultaneously handle both the importance and the influence of the elements in the decision system.

In the case of Yurihonjo, the author of this paper attempted to create an integrated procedure, which considers both the influence and the interdependencies of the elements and modifies the importance ranking based on these interdependencies. This is a novel approach and, although the first results are very promising, more tests are necessary to be able to state that a new methodology has been created.

\section{Methodology}

For the first AHP stage, questionnaires were made based on the hierarchical structure of the system elements as Figure 1 shows.

The results from the questionnaires are then aggregated into a matrix according to the following procedure described by Saaty (1977):

$$
f\left(y_{1}, \ldots, y_{l}\right)=\prod_{h=1}^{l}\left(y_{h}\right)^{1 / l},
$$




$$
\begin{aligned}
& l \geq 2, \\
& \left(y_{1}, \ldots, y_{l}\right) \in I^{l},
\end{aligned}
$$

where: $f$ is a function that summarizes the individual evaluations; $l$ is the number of evaluators. In addition, $y_{h}$ represents the properly indexed matrix element of the evaluator $h ; I^{l}$ is a set of positive numbers.

For determining the eigenvectors of the aggregate matrices, the following computation was applied:

$$
w_{r i}=\frac{w_{j}}{w}=\frac{w_{i j}}{\sum_{k=1}^{n} w_{i k}}=\left(\frac{w_{j}}{w} \cdot \frac{1}{\sum_{k=1}^{n} w_{i k}}\right) \cdot w_{i j},
$$

where: $j=1, \ldots, m ; w=\sum_{i=1}^{m} w_{j} ; w_{j}>0(j=1, \ldots, m)$ represents the related weight-coordinate from the previous level; $w_{i j}>0(i=1, \ldots, n)$ is the eigenvector computed from the matrix in the current level; $w_{r i}>0(i=1, \ldots, n)$ is the calculated weight score of the current level's elements.
When the eigenvectors are normalized, the following calculation can be executed:

$$
\begin{aligned}
& w_{r i}=w_{i j} \cdot w_{j}, \\
& i=1, \ldots, n,
\end{aligned}
$$

where: $w_{j}(j=1, \ldots, m)$ is the normalized weight of the previous level; $w_{i j}(i=1, \ldots, n)$ is the normalized eigenvectorcoordinate of the current level.

Therefore, in original AHP the resulting weights are normalized at the given levels, meaning that the derived weights should sum up 1 at the specific nests. In case of the AHP-ISM method the ultimate aim is to derive a final ranking of the different criteria throughout all the levels. Thus normalizing the weights at the specific nests is not the final step: to move towards final ranking, the normalized values are multiplied by the scores derived at the level above them, and this modifies the preference order at the given level, e.g. an element, which belongs to a branch of higher weights, will itself become more significant in the final ranking of the given level. Hence, the resulting values will not add up to 1 at the specific nests. Consequently,

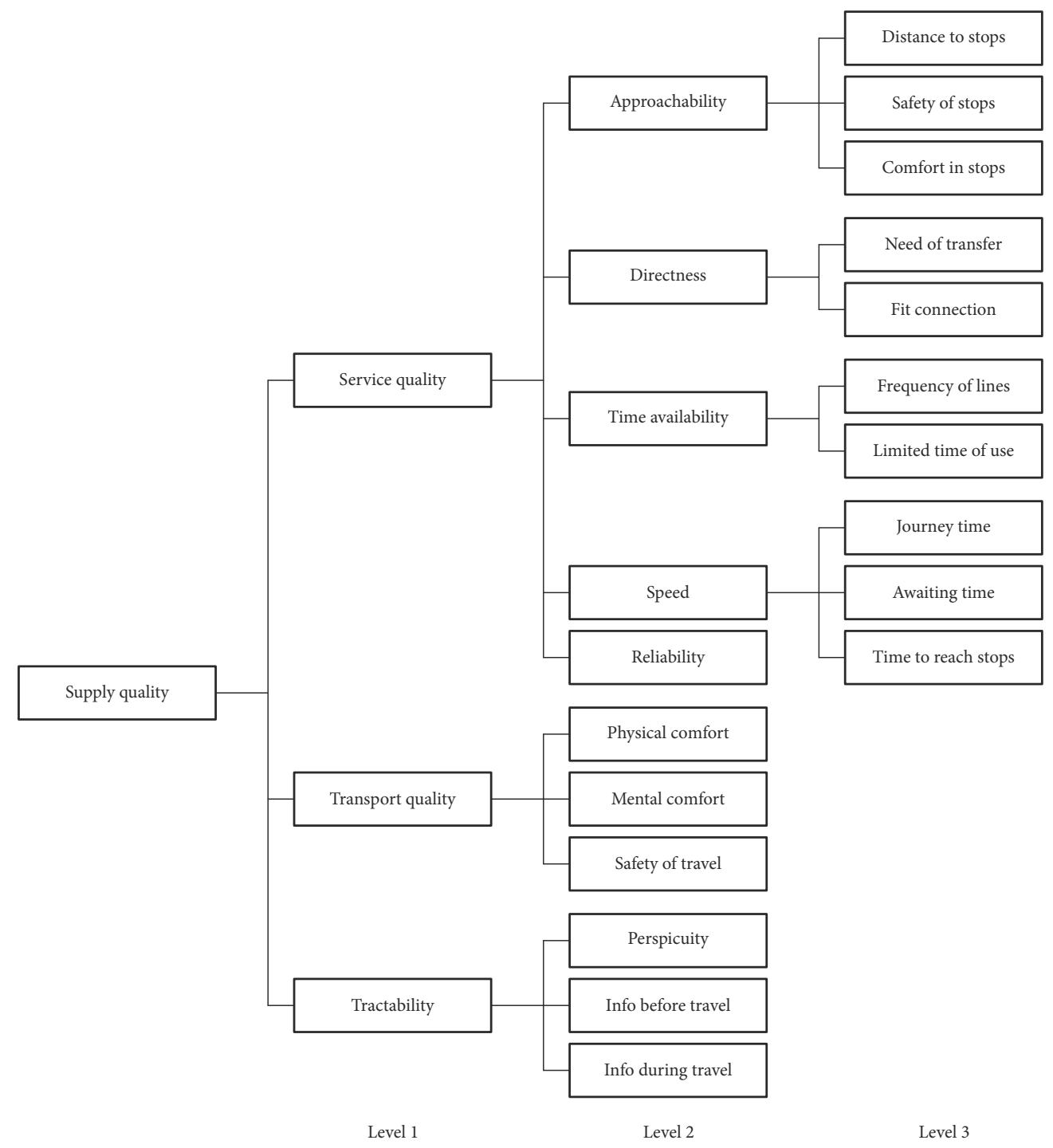

Figure 1. The hierarchical model of public bus transport supply quality (source: research based on Duleba et al. (2012)) 
the technique applied as the first step of the AHP-ISM method cannot be viewed as a traditional AHP, but only as a modified version of Saaty's methodology.

Consistency Ratio (CR) was also computed based on $C R=C I / R I$, where $C I=\frac{\lambda_{\max }-n}{n}-1$ is a Consistency Index; $R I$ is a Random Index depending on the size of the matrices. In our special case (Figure 1) $n=3(R I=0.58)$, $n=5(R I=1.12)$ and $n=2(R I$ does not exist) matrices had to be checked. It is a relevant debate among MCDM scientists, if $n=2$ matrices should be omitted or amended in AHP (Li et al. 2013; Saaty, Ozdemir 2003) in order to get complete consistency check, however the author of the recent paper decided to keep the $2 \times 2$ matrices (need of transfer-fit connection; frequency of lines-limited time of usage) because of their importance in the final problem and professional significance.

In order to establish the relationships, ISM constructs the so called Reachability Matrix (RM), which is a binary and quadratic matrix with rows and columns that are composed of system elements (for a system of $n$ elements, $\mathrm{RM}$ has a size of $n \times n)$. It is in this matrix that the existence of connections can be indicated. To state that a connection exists, the evaluators use the following principle:

$a_{i j}=1$, if element $i$ affects element $j$;

$a_{i j}=0$ in all other cases,

where: $e_{i}$ is the $i$-th element of the system; $a_{i j}$ denotes the relationship between the $i$-th and $j$-th elements.

What the evaluators do is filling up this matrix with $1 \mathrm{~s}$ and 0s, depending on whether the given element influences the other one, or not. Evidently, RM is not a symmetric matrix since the dependencies of the elements are not necessarily mutual. When used within the new AHP-ISM approach, ISM operates the following way: the hierarchical connections are copied from the previous, AHP-like step; placing $1 \mathrm{~s}$ at the connections where AHP hierarchy indicated a relationship. Then non-hierarchical linkages are established by the expert evaluators, and 1s are placed where a non-hierarchical connection is supposed to be exiting.

However, due to the restricted ability of the human mind, it is most likely that the evolving RM is not going to be transitive, meaning that respondents will not be always aware that if e.g. $e_{1}$ affects $e_{2}$, and $e_{2}$ affects $e_{3}$, then $e_{1}$ should influence $e_{3}$ as well, in a coherent fashion (i.e. if $a_{i j}=1$ and $a_{j k}=1$ then $a_{i k}=1$ ). This is especially true, when many elements are used. Thus, transitivity can be provided using the following matrix calculation:

$$
R M^{*}=R M^{c}=R M^{c+1},
$$

where: $c$ denotes the power; $R M^{*}$ is the final RM.

What Equation (4) does is multiplying the RM by itself as long as the evolving matrix becomes stabilized and does not change anymore. This is the point where transitivity is reached, and the resulting matrix, to be called the final RM, includes all the hierarchical and non-hierarchical connections in the system in a coherent way. To be able to attain this result, the final $\mathrm{RM}\left(R M^{\star}\right)$ has to be under the Boolean multiplication and addition operators (i.e., $1 \cdot 1=1$ and $1+1=1$ ). This is a necessary prerequisite to maintain the binarity of the final RM.

In the specific case of Yurihonjo project the value of $c$ was 3 , when the matrix became stable. The value of $c$ depends on the complexity of element interactions, the more complex the interdependency is, and the higher value of $c$ can be realized.

It has to be emphasized that ISM methodology is capable of determining reachability $R_{t i}$ and antecedent $A_{t i}$ sets and the graphical exhibition of these by using $R(t i) \cap A(t i)=R(t i)$ rules element by element. Nevertheless, for the introduced AHP-ISM procedure, Equation (4) provides the sufficient results that are applied in the next phase of the decision-making process.

By means of ISM, a map of all connections, both hierarchical and non-hierarchical, is achieved (for more detailed results, please see Duleba et al. (2013)). However, this information is not sufficient for modifying the AHP ranking, because further information is needed on the intensity of these connections.

In the previous stage, using ISM, non-hierarchical connections within the decision problem were established. Nonetheless, ISM is not capable of determining the strength of these connections. This is why the third stage is necessary, where relying on the results from ISM, the AHP scores are modified. This constitutes a new approach for combining the importance and influence of the elements. It shall be emphasized that there is a significant difference between the importance and influence of the elements: by definition, "importance" means the absolute merit gained by the given element. However, from a system-wide perspective it is easy to see, that if a certain element is improved, it does not only get better by itself, but it will also exert a positive influence on the criteria below it in the hierarchy. This effect is meant by the "influence" of the element. Combining "importance" and "influence" is done in the following way.

Let us denote:

$r_{i}$ - the $i$-th element of the system, $i=1, \ldots, n$;

$t_{r i}$ - all non-hierarchical connections of the element

$r_{i}, t=1, \ldots, s$;

$\mu_{t r i}$ - the specific rate of connection $t_{r i}$, which is determined by experts, $0<\left|\mu_{t r i}\right|<1$.

In addition, $\mu_{t r i}$ represents the rate of impact on the affected element, caused by improving the element $r_{i}$. Whereas $-r_{t r i}$ is the element that is affected by element $r_{i}$ in the connection of $t_{r i}$.

By using $\mu_{t r i}$, the original weight score of the element $r_{i}$ can be modified by adding the score of the affected element $r_{t r i}$ before multiplying by the rate of the connection (with $\mu_{t r i}$ ):

$$
w_{r i}^{\prime}=w_{r i}+\sum_{t=1}^{s} \mu_{t r i} \cdot w_{t r i}
$$

where: $w_{r i}^{\prime}$ denotes the modified score of element $r_{i}$ 
(a sum of all the "influences" on this element); $w_{r i}$ denotes the original AHP score of element $r_{i} ; \mu_{t r i}$ denotes the rate of (non-hierarchical) the connection $t$ (the "importance" of the element); $w_{t r i}$ denotes the score of the affected element by the impact of the connection $t$.

$w_{r i}$ and $w_{t r i}$ are original AHP scores of the elements. The AHP score of each element is modified by the nonhierarchical impacts originating from the other elements in the structure. The factors, which affect several other elements positively, will be accorded higher scores than the other elements. In addition, the value of $\mu_{t r i}$ may be negative if one of the elements affects the other negatively. In this case, the final score of that specific factor will decrease. Based on Equation (5), the importance and the influence of the factors within any general structure can be integrated in the decision-making process.

For example, if "frequency of lines" is improved in the system, so there would be more frequent bus lines, the "physical comfort" element is also improved due to the more space available for the passengers on a line. However, the improvement is not $100 \%$, but only $23 \%$ because there are more components of physical comfort not only the space for the passengers. In this case, the rate of this connection for element $r_{i}$ will be 0.23 , so $\mu_{k r i}=0.23$ if $k=1$ (in case it is the first non-hierarchical connection of "frequency of lines").

It has to be underlined that although these expert evaluations modify the original AHP scores gained by the passengers' opinions, this modification is indirect and only refer to additional points originated from the structural interrelationships of the factors within the system. Thus, passenger preferences can be kept and are only altered by additional expert knowledge on the structural linkages of the examined system.

\section{Results of the survey based on the created model}

Yurihonjo is a metropolitan area on the north-western coast of Japan, with a population of approximately 82000 inhabitants. 6 national highways and 2 railway lines cross the city, and it also disposes of a seaport. The last decade has seen a downward trend in the number of passengers opting for public. The decline in public transport participation might be due to changes of socio-economic or demographic factors, however based on passengers' feedback the dissatisfaction with the public transport service was obvious. This is the reason why the local government has decided to allocate funds to develop the public transport system and to do so with a view on the public opinion. This need was the main motivation behind the case study documented in the present section.

The case study followed the structure of the modified AHP-ISM approach detailed in the previous chapter. As a preliminary step, before the launch of the case study, the hierarchy of relationships was established by experts. Then, in the first stage a general AHP study was conducted eliciting the subjective opinion of 41 passengers. Sub- sequently, in the second stage, 3 experts determined the presence of non-hierarchical relationships via ISM. Finally, in the third stage, the knowledge gained was integrated into the AHP structure applying the novel approach explained above and introduced by the author. These steps are described further below.

\section{AHP stage}

As a prerequisite of research, a hierarchical model was created by experts for analysing passenger preferences for the city's public transportation system (Figure 1). Then, using this hierarchy, a sample consisting of 41 passengers evaluated the decision problem by the AHP questionnaire. Japanese middle-sized towns are characterized by the fact that the users of public transportation are dominantly either pensioners or students. This is even more relevant for Yurihinjo, where faculties of the Akita Prefectural University are settled, which attracts a significant proportion of the whole public transport demand within the town. The sample consisted of 28 university students and 13 pensioners, all active users of the public transportation system. After contacting the university students, they were asked to invite their grandparents who use city buses often and also former university administrational staff members were invited to participate in the evaluation process. Two instructors explained the AHP questionnaires and it took merely one hour to fill in the forms. Since the whole procedure (including the two other stages as well) is considered as decision support, statistical representativeness cannot be claimed.

Thus, this sample of respondents was invited to make pair-wise comparisons of the development alternatives level-wisely using the simplified Saaty-scale that was introduced in the previous section. After the consistency check (all resulted lower CR value than 0.1, the consistency threshold, most likely because of the participation of the instructors), the results were computed based on the passenger responses (Table 1, for further details and data of this stage the reader is referred to Duleba et al. (2012)). Nevertheless, it shall be noted here again, that the results of this stage do not match exactly those of a traditional AHP, as the ultimate aim is the ranking of all the criteria. Thus, the values in the different nests do not sum up 1, because they are already multiplied by the scores of the relevant superior branch.

As last part of the AHP phase, sensitivity analysis has been conducted, however, the classical AHP sensitivity analysis (Gao, Hailu 2012, 2013; Zhang et al. 2014) could not be applied due to the lack of alternatives. Instead, the Level 1. values had been altered and its impact was examined on the importance of lower level elements. The results showed stability, however, increasing the score of "Tractability" (and simultaneously decreasing "Service quality") caused rank reversal between "Info during" and "Approachability" factors, so sensitivity could be detected in this part of the hierarchy. 
Obviously, due to the application of the geometric mean (Equation (1)), the generalization of the results was made through the aggregation process. In case of 41 respondents, it is inevitable to detect some volatility among the evaluator scoring. For overcoming this problem, generally rank correlation methods are recommended e.g. Kendall rank correlation method. In the case of Yurihonjo, this analysis had not been selected because of two main reasons. Firstly (assumingly due to the work of instructors), there were no extreme differences and high volatility among the evaluations. Secondly, the aspect of rank correlation is ordinal, while the whole approach of Saaty's AHP (and the introduced AHP-ISM model in recent paper) is cardinal.

\section{ISM results}

In the second stage of the case study, three transportation experts from the local university evaluated the ISM RM and determined the existence of connections among the elements. It is normal to involve such a small sample of evaluators in an ISM application (Kannan et al. 2008; Iyer, Sagheer 2010). Thus, this stage was carried out by a small panel of experts, as expert knowledge is needed to reveal and pinpoint the underlying non-hierarchical connections, which are not always evident to laymen (e.g. average public transport users do not need to be aware of the fact that journey time might contribute to the safety of travel). The hierarchical connections were maintained from the previous stage of the study; only the non-hierarchical linkages were added in this phase.

Having conducted the ISM procedure, the following non-hierarchical connections (Table 2) could be determined. For additional details regarding this phase, please see Duleba et al. (2013).

\section{Results after modifying the AHP scoring}

In the modification phase, the numerical values of the hierarchical linkages were not altered, because both their existence and strength were determined in the AHP phase. Thus, in this stage their modification was not necessary. However, regarding the non-hierarchical connections, their strength was not established yet. In order to modify the relevant values, the strengths of these connections had to be determined to obtain the proper weights that affect the final scoring of the elements. As discussed above, it also had to be kept in mind that not only the "importance", but the "influence" of the elements also needed to be considered: i.e. an element that has a strong impact on other element/elements is more worthy of development than other elements.

Table 1. Results of the AHP phase (source: research by author)

\section{Level 1}

\begin{tabular}{|l|c|}
\hline Service quality & 0.456 \\
\hline Transport quality & 0.21 \\
\hline Tractability & 0.335 \\
\hline
\end{tabular}

\section{Level 2}

\begin{tabular}{|l|l|}
\hline Approachability & 0.147 \\
\hline Directness & 0.108 \\
\hline Time availability & 0.122 \\
\hline Speed & 0.094 \\
\hline Reliability & 0.072 \\
\hline
\end{tabular}

Ranking
\begin{tabular}{|c|l|c|}
\hline 1 & Service quality & 0.456 \\
\hline 2 & Tractability & 0.335 \\
\hline 3 & Transport quality & 0.21 \\
\hline
\end{tabular}

Ranking

\begin{tabular}{|l|l|}
\hline Physical comfort & 0.033 \\
\hline Mental comfort & 0.093 \\
\hline Safety of travel & 0.084 \\
\hline
\end{tabular} \begin{tabular}{|l|l|}
\hline Perspicuity & 0.122 \\
\hline Info before & 0.082 \\
\hline Info during & 0.131 \\
\hline
\end{tabular}

\begin{tabular}{|c|l|c|}
\hline 1 & Approachability & 0.147 \\
\hline 2 & Info during & 0.131 \\
\hline 3 & Perspicuity & 0.122 \\
\hline 3 & Time availability & 0.122 \\
\hline 5 & Directness & 0.108 \\
\hline 6 & Speed & 0.094 \\
\hline 7 & Mental comfort & 0.093 \\
\hline 8 & Safety of travel & 0.084 \\
\hline 9 & Info before & 0.082 \\
\hline 10 & Reliability & 0.072 \\
\hline 11 & Physical comfort & 0.033 \\
\hline
\end{tabular}

Ranking

Level 3

\begin{tabular}{|l|c|}
\hline Distance to stops & 0.071 \\
\hline Safety of stops & 0.037 \\
\hline Comfort in stops & 0.04 \\
\hline
\end{tabular}

\begin{tabular}{|l|c|}
\hline Need of transfer & 0.058 \\
\hline Fit connection & 0.05 \\
\hline
\end{tabular}

\begin{tabular}{|l|l|}
\hline Frequency of lines & 0.092 \\
\hline Limited time of usage & 0.030 \\
\hline
\end{tabular}

\begin{tabular}{|l|l|}
\hline Journey time & 0.053 \\
\hline Awaiting time & 0.016 \\
\hline Time to reach stops & 0.025 \\
\hline
\end{tabular}

\begin{tabular}{|c|l|c|}
\hline 1 & Frequency of lines & 0.092 \\
\hline 2 & Distance to stops & 0.071 \\
\hline 3 & Need of transfer & 0.058 \\
\hline 4 & Journey time & 0.053 \\
\hline 5 & Fit connection & 0.05 \\
\hline 6 & Comfort in stops & 0.04 \\
\hline 7 & Safety of stops & 0.037 \\
\hline 8 & Limited time of usage & 0.03 \\
\hline 9 & Time to reach stops & 0.025 \\
\hline 10 & Awaiting time & 0.016 \\
\hline
\end{tabular}


The numerical values of connection strengths were determined by expert evaluation (Table 3): sixteen transportation experts (including professors from transport science faculties at international universities and public transportation company managers) were asked to evaluate the strength of each connection using a number between 0 and 1. It was assumed that all non-hierarchical linkage weights were between 0 and 1 (while hierarchical weights equalled 1 , as these did not need to be changed by the expert evaluation). In this way, they assigned an estimated value to every non-hierarchical connection.

The strengths of these connections are system-specific. For example, the linkage of "frequency of lines" and "physical comfort" has a weak connection if public vehicles are not used frequently. However, dependency is strong when buses are frequently utilized. It shall be noted that this dependency was relatively low in Yurihonjo. These specificities need to be considered by the experts when establishing the strength of these relationships.

The scores are used in the further calculations to modify the final full ranking of the criteria.

Using these scores describing the non-hierarchical connections $w_{t r i}$ and the numerical values of the hierarchical connections from Step $1 w_{r i}$, the final modified scores and rankings can be established by Equation (5). The evolving modified scores and rankings are listed in Table 4, which presents the ultimate results of the new AHP-ISM approach: a final, full ranking of the criteria, which can be taken into consideration in public transport system redesign.

As a summary, the following exhibit makes the integrated data collection and procedure visible (Figure 2).

\section{Conclusions}

When creating transportation system management strategies, the recent decades have shown how important it is to consider the users' preferences, even if they lack technological or economic aspects. The overall aim to motivate the shift from car use to public transportation can only be reached if the public transportation system meets the requirements of potential passengers. Therefore, better decisions can be made for public transportation when more applicable and sufficient information is obtained.

Table 2. Interrelations of the factors based on ISM results (source: research by author)

\begin{tabular}{|l|l|}
\hline \multicolumn{1}{|c|}{ Driver element } & \multicolumn{1}{c|}{ Affected elements } \\
\hline directness & tractability; speed; reliability; info before; fit connection; awaiting time \\
\hline time average & speed \\
\hline reliability & tractability; directness; speed; info before; fit connection; awaiting time \\
\hline perspicuity & service quality; speed; awaiting time \\
\hline info before & service quality; speed; awaiting time \\
\hline info during & transport quality; mental comfort \\
\hline distance to stops & speed; time to reach stops \\
\hline need of transfer & tractability; speed; reliability; info before; fit connection; awaiting time \\
\hline fit connection & tractability; speed; reliability; info before; awaiting time \\
\hline frequency of lines & transport quality; speed; physical comfort; awaiting time \\
\hline limited time of usage & tractability; speed; info before; awaiting time \\
\hline journey time & transport quality; physical comfort; mental comfort; safety of travel \\
\hline
\end{tabular}

Table 3. Interrelations of the factors regarding the strength of the connection (source: research by author)

\begin{tabular}{|c|c|}
\hline Driver element & Affected elements \\
\hline directness & tractability 0.16 ; speed 0.72 ; reliability 0.64 ; info before 0.62 ; fit connection 0.41 ; awaiting time 0.74 \\
\hline time average & speed 0.39 \\
\hline reliability & tractability 0.31 ; directness 0.21 ; speed 0.62 ; info before 0.23 ; fit connection 0.92 ; awaiting time 0.81 \\
\hline perspicuity & service quality 0.12 ; speed 0.23 ; awaiting time 0.63 \\
\hline info before & service quality 0.13 ; speed 0.24 ; awaiting time 0,72 \\
\hline info during & transport quality 0.32 ; mental comfort 0.53 \\
\hline distance to stops & speed $0.24 ;$ time to reach stops 0.92 \\
\hline need of transfer & tractability 0.31 ; speed 0.81 ; reliability 0.68 ; info before 0.68 ; fit connection 0.83 ; awaiting time 0.63 \\
\hline fit connection & tractability 0.19 ; speed 0.61 ; reliability 0.72 ; info before 0.48 ; awaiting time 0.71 \\
\hline frequency of lines & transport quality 0.25 ; speed 0.87 ; physical comfort 0.17 ; awaiting time 0.52 \\
\hline limited time of usage & tractability 0.19 ; speed 0.32 ; info before 0.57 ; awaiting time 0.34 \\
\hline journey time & transport quality 0.21 ; physical comfort 0.52 ; mental comfort 0.54 ; safety of travel 0.52 \\
\hline
\end{tabular}


Table 4. The ultimate modified scores of the integrated approach AHP-ISM (source: research by author)

Level 1

\begin{tabular}{|l|c|}
\hline Service quality & 0.456 \\
\hline Transport quality & 0.21 \\
\hline Tractability & 0.335 \\
\hline
\end{tabular}

Level 2

\begin{tabular}{|l|c|}
\hline Approachability & 0.147 \\
\hline Directness & 0.34 \\
\hline Time availability & 0.169 \\
\hline Speed & 0.094 \\
\hline Reliability & 0.306 \\
\hline
\end{tabular}

\begin{tabular}{|l|l|}
\hline Physical comfort & 0.033 \\
\hline Mental comfort & 0.093 \\
\hline Safety of travel & 0.084 \\
\hline
\end{tabular}

\begin{tabular}{|l|l|}
\hline Perspicuity & 0.207 \\
\hline Info before & 0.157 \\
\hline Info during & 0.324 \\
\hline
\end{tabular}

Level 3

\begin{tabular}{|l|c|}
\hline Distance to stops & 0.071 \\
\hline Safety of stops & 0.037 \\
\hline Comfort in stops & 0.04 \\
\hline
\end{tabular}

\begin{tabular}{|l|l|}
\hline Need of transfer & 0.391 \\
\hline Fit connection & 0.257 \\
\hline
\end{tabular}

\begin{tabular}{|l|l|}
\hline Frequency of lines & 0.226 \\
\hline Limited time of usage & 0.175 \\
\hline
\end{tabular}

\begin{tabular}{|l|l|}
\hline Journey time & 0.217 \\
\hline Awaiting time & 0.016 \\
\hline Time to reach stops & 0.025 \\
\hline
\end{tabular}

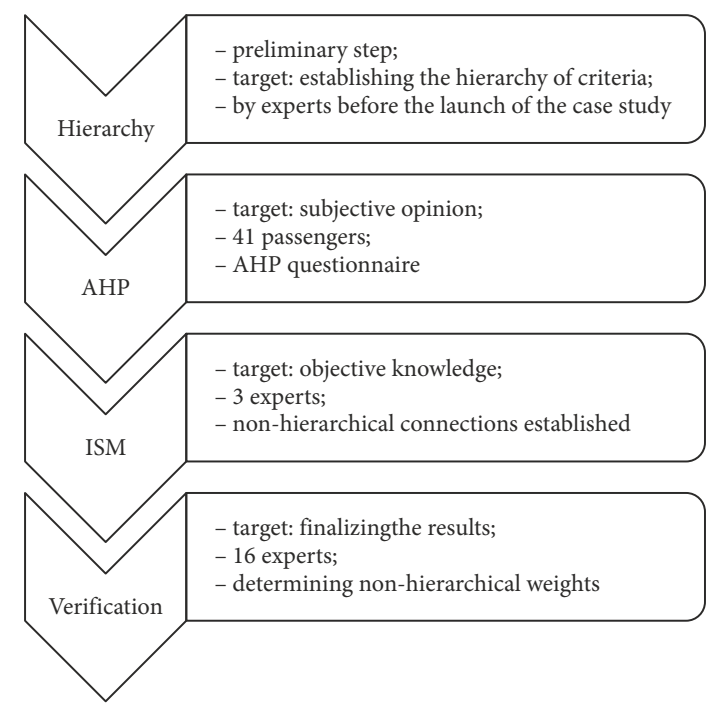

Figure 2. Description of the AHP-ISM procedure (source: research by author)

In the concrete example the analysis revealed that the most important and influential factor that should be improved in Yurihonjo's public transport system from the passengers' perspective is the "need of transfer" factor.
Ranking

\begin{tabular}{|c|l|c|}
\hline 1 & Service quality & 0.456 \\
\hline 2 & Tractability & 0.335 \\
\hline 3 & Transport quality & 0.21 \\
\hline
\end{tabular}

Ranking

\begin{tabular}{|c|l|c|}
\hline 1 & Directness & 0.34 \\
\hline 2 & Info during & 0.324 \\
\hline 3 & Reliability & 0.306 \\
\hline 4 & Perspicuity & 0.207 \\
\hline 5 & Time availability & 0.169 \\
\hline 6 & Info before & 0.157 \\
\hline 7 & Approachability & 0.147 \\
\hline 8 & Speed & 0.094 \\
\hline 9 & Mental comfort & 0.093 \\
\hline 10 & Safety of travel & 0.084 \\
\hline 11 & Physical comfort & 0.033 \\
\hline
\end{tabular}

Ranking

\begin{tabular}{|c|l|c|}
\hline 1 & Need of transfer & 0.391 \\
\hline 2 & Fit connection & 0.257 \\
\hline 3 & Frequency of lines & 0.226 \\
\hline 4 & Journey time & 0.217 \\
\hline 5 & Limited time of usage & 0.175 \\
\hline 6 & Distance to stops & 0.071 \\
\hline 7 & Comfort in stops & 0.04 \\
\hline 8 & Safety of stops & 0.037 \\
\hline 9 & Time to reach stops & 0.025 \\
\hline 10 & Awaiting time & 0.016 \\
\hline
\end{tabular}

In addition, the "fit connection" and "frequency of lines" factors also proved to be significant. Thus, development projects should aim to improve these elements to enhance the service quality of the system.

Nevertheless, the limits of the introduced technique shall be highlighted as well. For the first stage, recruiting passenger evaluators for AHP procedure is difficult and due to the relatively complex questionnaire, all support has to be given to the participants. Another limitation of the new procedure is that the results are not tied to the specific lines of the system, more specification is needed for determining development claims for specific lines of the public transport system.

The local government of the town decided to implement the results to public transport strategy, which hints the potential applicability of the introduced procedure. For European municipalities, in the process of creating their Sustainable Urban Mobility Plans (SUMP), this AHP-ISM methodology might be a very useful tool for embedding public needs in strategic improvement planning.

Further potential for research is to integrate the opinions of different evaluator groups' opinions, e.g., users, company managers, and governmental officers (i.e. allimportant stakeholders of public transportation systems). 
Creating a final consensus on improvement issues and considering the importance and influences of the factors will prove to be useful for ranking different transport projects.

\section{Funding}

The author would like to acknowledge the financial support of the PIAC_13-1-2013-0176 research program.

\section{Disclosure statement}

The author states that he does not have any competing financial, professional, or personal interests from other parties.

\section{References}

Aparicio, A. 2007. Assessing public involvement effectiveness in long-term planning, in Transportation Research Board 86th Annual Meeting, 21-25 January 2007, Washington, DC, US, $1-9$.

Al-Atawi, A. M.; Kumar, R.; Saleh, W. 2016. Transportation sustainability index for Tabuk city in Saudi Arabia: an analytic hierarchy process, Transport 31(1): 47-55. https://doi.org/10.3846/16484142.2015.1058857

Barić, D.; Pilko, H.; Strujić, J. 2016. An analytic hierarchy process model to evaluate road section design, Transport 31(3): 312-321. https://doi.org/10.3846/16484142.2016.1157830

Bhushan, N.; Ria, K. 2004. Strategic Decision Making: Applying the Analytic Hierarchy Process. Springer-Verlag London. $172 \mathrm{p}$.

Burall, S.; Shahrokh, T. 2010. What the Public Say: Public Engagement in National Decision-Making. Involve Company, UK. 18 p. Available from Internet: https://www.involve.org. uk/resources/publications/project-reports/what-public-saypublic-engagement-national-decision-making

Cascetta, E.; Pagliara, F. 2013. Public engagement for planning and designing transportation systems, Procedia - Social and Behavioral Sciences 87: 103-116.

https://doi.org/10.1016/j.sbspro.2013.10.597

Chen, S.; Pham, V. K.; Chen, J. K. 2016. Evaluating and selecting the best outsourcing service country in East and Southeast Asia: an AHP approach, Journal of Testing and Evaluation 44(1): 89-101. https://doi.org/10.1520/JTE20140065

De Luca, S. 2014. Public engagement in strategic transportation planning: an analytic hierarchy process based approach, Transport Policy 33: 110-124. https://doi.org/10.1016/j.tranpol.2014.03.002

Duleba, S.; Mishina, T.; Shimazaki, Y. 2012. A dynamic analysis on public bus transport's supply quality by using AHP, Transport 27(3): 268-275. https://doi.org/10.3846/16484142.2012.719838

Duleba, S.; Shimazaki, Y.; Mishina, T. 2013. An analysis on the connections of factors in a public transport system by AHPISM, Transport 28(4): 404-412.

https://doi.org/10.3846/16484142.2013.867282

Eswarlal, V. K.; Dey, P. K.; Shankar, R. 2011. Enhanced renewable energy adoption for sustainable development in India: interpretive structural modeling approach, in Proceedings from the World Renewable Energy Congress, 8-13 May 2011, Linkoping, Sweden, 351-358. https://doi.org/10.3384/ecp11057351
Gao, L.; Hailu, A. 2013. Identifying preferred management options: an integrated agent-based recreational fishing simulation model with an AHP-TOPSIS evaluation method, Ecological Modelling 249: 75-83.

https://doi.org/10.1016/j.ecolmodel.2012.07.002

Gao, L.; Hailu, A. 2012. Ranking management strategies with complex outcomes: an AHP-fuzzy evaluation of recreational fishing using an integrated agent-based model of a coral reef ecosystem, Environmental Modelling \& Software 31: 3-18. https://doi.org/10.1016/j.envsoft.2011.12.002

Iyer, K. C.; Sagheer, M. 2010. Hierarchical structuring of PPP risks using interpretative structural modeling, Journal of Construction Engineering and Management 136(2): 151-159. https://doi.org/10.1061/(ASCE)CO.1943-7862.0000127

Jen, W.; Tu, R.; Lu, T. 2011. Managing passenger behavioral intention: an integrated framework for service quality, satisfaction, perceived value, and switching barriers, Transportation 38(2): 321-342. https://doi.org/10.1007/s11116-010-9306-9

Jharkharia, S.; Shankar, R. 2007. Selection of logistics service provider: an analytic network process (ANP) approach, Omega 35(3): 274-289. https://doi.org/10.1016/j.omega.2005.06.005

Kannan, G.; Noorul Haq, A.; Sasikumar, P.; Arunachalam, S. 2008. Analysis and selection of green suppliers using interpretative structural modelling and analytic hierarchy process, International Journal of Management and Decision Making 9(2): 163-182. https://doi.org/10.1504/IJMDM.2008.017198

Li, F.; Phoon, K. K.; Du, X.; Zhang, M. 2013. Improved AHP method and its application in risk identification, Journal of Construction Engineering and Management 139(3): 312-320. https://doi.org/10.1061/(ASCE)CO.1943-7862.0000605

Magginas, V.; Nathanail, E.; Manoli, S.; Malnaca, K. 2018. A multi-agent approach towards designing a city port business model, Transport and Telecommunication 19(3): 213-223. https://doi.org/10.2478/ttj-2018-0018

Malone, D. W. 1975. An introduction to the application of interpretive structural modeling, Proceedings of the IEEE 63(3): 397-404. https://doi.org/10.1109/PROC.1975.9765

Pfohl, H.-C.; Gallus, P.; Thomas, D. 2011. Interpretive structural modeling of supply chain risks, International Journal of Physical Distribution \& Logistics Management 41(9): 839-859. https://doi.org/10.1108/09600031111175816

Renn, O.; Webler, T.; Wiedemann, P. 1995. Fairness and Competence in Citizen Participation: Evaluating Models for Environmental Discourse. Springer Netherlands. 381 p. https://doi.org/10.1007/978-94-011-0131-8

Saaty, T. L. 1977. A scaling method for priorities in hierarchical structures, Journal of Mathematical Psychology 15(3): 234281. https://doi.org/10.1016/0022-2496(77)90033-5

Saaty, T. L. 2013. Analytic network process, in S. I. Gass, M. C. Fu (Eds.). Encyclopedia of Operations Research and Management Science. https://doi.org/10.1007/978-1-4419-1153-7_32

Saaty, T. L. 2001. The Analytic Network Process: Decision Making with Dependence and Feedback. RWS publications. 370 p.

Saaty, T. L.; Ozdemir, M. S. 2003. Why the magic number seven plus or minus two, Mathematical and Computer Modelling 38(3-4): 233-244.

https://doi.org/10.1016/S0895-7177(03)90083-5

Saleeshya, P. G.; Thampi, K. S.; Raghuram, P. 2012. A combined AHP and ISM-based model to assess the agility of supply chain - a case study, International Journal of Integrated Supply Management 7(1-3): 167-191.

https://doi.org/10.1504/IJISM.2012.051050 
Tudela, A.; Akiki, N.; Cisternas, R. 2006. Comparing the output of cost benefit and multi-criteria analysis: An application to urban transport investments, Transportation Research Part A: Policy and Practice 40(5): 414-423.

https://doi.org/10.1016/j.tra.2005.08.002

US DoT. 2015. A Guide to Transportation Decisionmaking. 24 p. US Department of Transportation (DoT), Washington, DC, US. Available from Internet: https://www.planning.dot.gov/ documents/GuidetoTransportationDecisionmaking.pdf

US Government. 2005. Safe, Accountable, Flexible, Efficient Transportation Equity Act: a Legacy for Users. Public Law 109-59. 10 August 2005. US Government Printing Office. 836 p. Available from Internet: https://www.govinfo.gov/content/pkg/PLAW-109publ59/pdf/PLAW-109publ59.pdf

Vitić-Ćetković, A.; Bauk, S. 2014. E-services and positioning of passenger ports in the context of cruise tourism promotion, Promet - Traffic \& Transportation 26(1): 83-93. https://doi.org/10.7307/ptt.v26i1.1282

Yedla, S.; Shrestha, R. M. 2003. Multi-criteria approach for the selection of alternative options for environmentally sustainable transport system in Delhi, Transportation Research Part A: Policy and Practice 37(8): 717-729. https://doi.org/10.1016/S0965-8564(03)00027-2

Zhang, X.; Gao, L.; Barrett, D.; Chen, Y. 2014. Evaluating water management practice for sustainable mining, Water 6(2): 414-433. https://doi.org/10.3390/w6020414 